\title{
VOLTERRA INTEGRAL EQUATIONS ON VARIABLE EXPONENT LEBESGUE SPACES
}

\author{
R.E. CASTILLO, J.C. RAMOS-FERNÁNDEZ AND E.M. ROJAS
}

Communicated by Hermann Brunner

\begin{abstract}
In this paper, in the framework of Lebesgue spaces with variable exponent, we are going to provide conditions for the existence and uniqueness of the solutions of a class of Volterra integral equations induced by Carathéodory functions having diverse growth behaviors. To attain our goals, we will use topological degree theory for condensing maps and fixed point results for the sum of mappings of contractive type.
\end{abstract}

1. Introduction. Existence, uniqueness and stability of solutions, as well as the numerical aspect of Volterra integral equations have been widely studied in the literature. Part of this interest arises from the vast range of applications where this kind of equations appears, for instance in semiconductors, fluid flow, chemical reactions, elasticity and population dynamics, among others. More information can be found in the monographs $[\mathbf{1}, \mathbf{2}, \mathbf{3}, \mathbf{1 5}, \mathbf{2 2}]$ and in references on the subject. Typically, these equations have been studied on Lebesgue spaces and the space of continuous functions (real, complex or Banach spaces valued), even though, due to its role in applications to spectral theory, integral and differential equations and embeddings of Sobolev spaces, they also have been considered on the framework of Sobolev spaces [12, 21, 23].

However, in recent years, it became clear that the classical function spaces are not sufficient for solving the contemporary problems of nonlinear elasticity, in modeling of various fluids flows, current variation

2010 AMS Mathematics subject classification. Primary 45D05, 46E30, 47B38, $47 \mathrm{H} 10$.

Keywords and phrases. Volterra integral equation, variable Lebesgue space, contractive mapping.

Received by the editors on April 12, 2015, and in revised form on August 8, 2015. 
and problems of mechanics. Nevertheless, the theory of spaces of functions with nonstandard growth, also known as generalized Lebesgue spaces with variable exponent, have been extensively studied in recent years (see, e.g., $[\mathbf{4}, \mathbf{5}, \mathbf{1 0}]$ and references therein). These spaces arise in applications to continuum mechanics (in particular, in the theory of electrorheological fluids). They also arise in the theory of differential equations and variational problems. A lot of the results in this line of research are condensed in the monographs $[\mathbf{1 0}, \mathbf{3 2}]$.

Thus, due to the wide range of applications where Lebesgue spaces with variable exponent can be used to overcome the limitations of the classical spaces, in this paper we will analyze the existence and uniqueness of the solutions of a type of Volterra integral equation in this context (see, equation (3.1) below). More precisely, we are going to consider integral equations induced by Carathéodory functions having different growth behaviors. The techniques that we will use here are the classical topological degree theory and the new setting of sum of contractive type of mappings. We would like to point out that, in the most abstract scheme of Banach function spaces, the generalized Hardy operator, which is a Volterra-type operator, is studied in [14].

The paper is divided into four sections. In Section 2, we will present the preliminary results on variable exponent Lebesgue spaces, degree theory for condensing maps and sum of contractive mappings, which will be useful in the sequel. Section 3 is devoted to studying the continuity boundedness and compactness of the operators associated to the equations considered here. Results regarding the existence and uniqueness of the solutions of equation (3.1) will be presented in Section 4.

2. Preliminaries. In this section, we are going to give preliminary results which will be useful in the sequel.

2.1. Variable exponent Lebesgue spaces. We now review some definitions and properties related to Lebesgue spaces with variable exponents needed throughout the paper. For a more comprehensive study of these spaces we recommend the monographs $[\mathbf{4}, \mathbf{1 0}]$.

Let $\Omega \subseteq \mathbb{R}^{n}$ be an open set in $\mathbb{R}^{n}$. By $L^{p(\cdot)}(\Omega)$, we denote the space of functions on $\Omega$ such that 


$$
\rho_{p(\cdot)}(f):=\int_{\Omega}|f(x)|^{p(x)} \mathrm{d} x<\infty
$$

where $p(\cdot)$ is a measurable function on $\Omega$ with values in $[1, \infty)$ and define $p_{-}:=\operatorname{essinf}{ }_{x \in \Omega} p(x)$ and $p_{+}:=\operatorname{ess} \sup _{x \in \Omega} p(x)$. The modular functional $\rho_{p(\cdot)}$ satisfies the following fundamental properties.

Proposition 2.1. Given $\Omega$ and $p(\cdot)$ a measurable function on $\Omega$ :

(i) For all $f, \rho_{p(\cdot)}(f) \geq 0$ and $\rho_{p(\cdot)}(|f|)=\rho_{p(\cdot)}(f)$.

(ii) $\rho_{p(\cdot)}(f)=0$ if and only if $f(x)=0$ for almost every $x \in \Omega$.

(iii) If $\rho_{p(\cdot)}(f)<\infty$, then $f(x)<\infty$ for almost every $x \in \Omega$.

(iv) $\rho_{p(\cdot)}$ is order preserving: if $|f(x)| \geq|g(x)|$ almost everywhere, then $\rho_{p(\cdot)}(f) \geq \rho_{p(\cdot)}(g)$.

The functional $\rho_{p(\cdot)}$ does not satisfy the triangle inequality; however, if $p_{+}<\infty$, the modular triangle inequality holds:

$$
\rho_{p(\cdot)}(f+g) \leq 2^{p_{+}}\left(\rho_{p(\cdot)}(f)+\rho_{p(\cdot)}(g)\right) .
$$

$L^{p(\cdot)}(\Omega)$ becomes a Banach space with the norm (the so-called Kolmogorov-Minkowski norm)

$$
\|f\|_{p(\cdot)}:=\inf \left\{\lambda>0: \rho_{p(\cdot)}\left(\frac{f}{\lambda}\right) \leq 1\right\} .
$$

If $p(\cdot)$ satisfies that

$$
1<p_{-}, \quad p_{+}<\infty
$$

then the function $p^{\prime}(x):=p(x) /(p(x)-1)$ is well defined, satisfies (2.1) and

$$
\frac{1}{p(x)}+\frac{1}{p^{\prime}(x)}=1 \text {. }
$$

Moreover, if $p_{+}<\infty$, then the set of all continuous functions whose support is compact and contained in $\Omega$ is dense in $L^{p(\cdot)}(\Omega)$.

The following result shows a relation between the modular functional $\rho_{p(\cdot)}(\cdot)$ and the norm $\|\cdot\|_{p(\cdot)}$. In particular, this shows that in matters of convergence both notions are equivalent. 
Proposition 2.2. For any function $p(\cdot), 1 \leq p_{-} \leq p(x) \leq p_{+}<\infty$ almost everywhere in $\Omega$ we have

$$
\|f\|_{p(\cdot)}^{p_{+}} \leq \rho_{p(\cdot)}(f) \leq\|f\|_{p(\cdot)}^{p_{-}}, \quad \text { if }\|f\|_{p(\cdot)} \leq 1,
$$

the signs of the inequality being opposite if $\|f\|_{p(\cdot)} \geq 1$.

Lemma 2.3. Let $0<\gamma(x) \leq p(x) \leq p_{+}<\infty, x \in \Omega$. Then

$$
\|f\|_{p(\cdot)}^{\gamma_{+}} \leq\left\|f^{\gamma}\right\|_{p(\cdot) / \gamma(\cdot)} \leq\|f\|_{p(\cdot)}^{\gamma_{-}}, \quad \text { if }\|f\|_{p(\cdot)} \leq 1,
$$

where $f^{\gamma}:=|f(x)|^{\gamma(x)}$, the signs of the inequality being opposite if $\|f\|_{p(\cdot)} \geq 1$.

In this paper, we will deal with sets $\Omega$ with finite Lebesgue measure. Thus, under this condition, the function $1 \in L^{p(\cdot)}(\Omega)$. Also, in this case, the embedding characterization of variable Lebesgue spaces into one another is: Given $p(\cdot)$ and $q(\cdot)$, then $L^{q(\cdot)}(\Omega) \hookrightarrow L^{p(\cdot)}(\Omega)$ if and only if $p(x) \leq q(x)$ almost everywhere $x \in \Omega$ (see [4, page 41, Corollary $2.48])$.

The Hölder inequality in this space is given in the form: for $f \in$ $L^{p(\cdot)}(\Omega), g \in L^{p^{\prime}(\cdot)}(\Omega)$ with $1 \leq p(x) \leq \infty$,

$$
\int_{\Omega}|f(x) g(x)| \mathrm{d} x \leq K_{p(\cdot)}\|f\|_{p(\cdot)}\|g\|_{p^{\prime}(x)},
$$

where $K_{p(\cdot)}:=1 / p_{-}+1 / p_{-}^{\prime}=\sup 1 / p(x)+\sup 1 / p^{\prime}(x)$.

The Minkowski integral inequality is given by: for $1 \leq p(x) \leq p_{+}<$ $\infty$, let $f: \Omega \times \Omega \longrightarrow \mathbb{R}$ be a measurable function with respect to the product measure such that almost everywhere $y \in \Omega$, we have $f(\cdot, y) \in L^{p(\cdot)}(\Omega)$. Then

$$
\left\|\int_{\Omega} f(\cdot, y) \mathrm{d} y\right\|_{p(\cdot)} \leq 2 K_{p(\cdot)} \int_{\Omega}\|f(\cdot, y)\|_{p(\cdot)} \mathrm{d} y .
$$

Proposition 2.4. (See [11, Remark 2.1]). Suppose the sequence $\left\{f_{k}\right\} \subset L^{p(\cdot)}(\Omega)$ converges in norm to $f \in L^{p(\cdot)}(\Omega)$. Then there exists a subsequence $\left\{f_{k_{j}}\right\}$ and $g \in L^{p(\cdot)}(\Omega)$ such that the subsequence converges pointwise almost everywhere to $f$, and, for almost every $x \in \Omega,\left|f_{k_{j}}(x)\right| \leq g(x)$. 
On the other hand, let $g(x, u),(x \in \Omega, u \in \mathbb{R})$ be a Carathéodory function. The boundedness of the Nemytsky operator defined by $g(x, u)$

$$
(G u)(x)=g(x, u(x))
$$

in the framework of variable exponent Lebesgue spaces is given in the following result:

Theorem 2.5 ([13]). If $G$ maps $L^{p(\cdot)}(\Omega)$ into $L^{q(\cdot)}(\Omega)$, then $G$ is continuous and bounded, and there is a constant $b \geq 0$ and a nonnegative function $a \in L^{q(\cdot)}(\Omega)$ such that, for $x \in \Omega$ and $u \in \mathbb{R}$, the following inequality holds:

$$
|g(x, u)| \leq a(x)+b|u|^{p(x) / q(x)} .
$$

On the other hand, if $g(x, u)$ satisfies $(2.2)$, then $G$ maps $L^{p(\cdot)}(\Omega)$ into $L^{q(\cdot)}(\Omega)$, and thus $G$ is continuous and bounded.

2.2. Degree theory for condensing maps. In order to prove some of our results, we will study $\alpha$-set contractions and condensing maps. For more information about this we refer the readers to [6].

Let $X$ be a Banach space and $\mathcal{B} \subset \mathcal{P}(X)$ the family of all its bounded sets.

The (Kuratowski-) measure of noncompactness is defined by the function $\alpha: \mathcal{B} \rightarrow \mathbb{R}_{+}$given, for each $B \in \mathcal{B}$, as

$$
\alpha(B)=\inf \{d>0: B \text { admits a finite cover by set of diameter } \leq d\} .
$$

The function $\alpha$ satisfies the following properties.

Proposition 2.6. The following assertions hold:

(i) $\alpha(B)=0$ if and only if $B$ is relatively compact.

(ii) $\alpha$ is a seminorm, i.e., $\alpha(\lambda B)=|\lambda| \alpha(B)$ and $\alpha\left(B_{1}+B_{2}\right) \leq$ $\alpha\left(B_{1}\right)+\alpha\left(B_{2}\right)$.

(iii) $B_{1} \subset B_{2}$ implies $\alpha\left(B_{1}\right) \leq \alpha\left(B_{2}\right)$.

(iv) $\alpha\left(B_{1} \cup B_{2}\right)=\max \left\{\alpha\left(B_{1}\right), \alpha\left(B_{2}\right)\right\}$.

(v) $\alpha(\operatorname{conv} B)=\alpha(B)$.

(vi) $\alpha(\bar{B})=\alpha(B)$. 
Let $\Omega \subset X$ and $F: \Omega \rightarrow X$ be a continuous bounded map. $F$ is called $\alpha$-Lipschitz if there exists $k \geq 0$ such that

$$
\alpha(F(B)) \leq k \alpha(B), \quad \text { for all } B \subset \Omega \text { bounded } .
$$

If, in addition, $k<1$, then we say that $F$ is strict $\alpha$-contraction. The map $F$ is $\alpha$-condensing if

$$
\alpha(F(B))<\alpha(B), \quad \text { for all } B \subset \Omega \text { bounded with } \alpha(B)>0 .
$$

Proposition 2.7. The following assertions hold:

(i) If F, $G: \Omega \rightarrow X$ are $\alpha$-Lipschitz maps with constants $k_{1}$ and $k_{2}$, respectively, then $F+G: \Omega \rightarrow X$ is $\alpha$-Lipschitz with constant $k_{1}+k_{2}$.

(ii) If $F: \Omega \rightarrow X$ is compact, then $F$ is $\alpha$-Lipschitz with constant $k=0$.

(iii) If $F: \Omega \rightarrow X$ is a Lipschitz map with constant $k$, then $F$ is $\alpha$-Lipschitz with the same constant $k$.

Using the basic properties of the topological degree for $\alpha$-condensing perturbation of the identity, in [17] the following fixed point result was proved.

Theorem 2.8. Let $F: X \rightarrow X$ be $\alpha$-condensing and

$$
S=\{x \in X: \text { there exists } \lambda \in[0,1] \text { such that } x=\lambda F x\} .
$$

If $S$ is a bounded set in $X$, then there exists an $r>0$ such that $S \subset B_{X}(r)$, and $F$ has at least one fixed point. The set of fixed points of $F$ lies in $B_{X}(r)$, where $B_{X}(r)$ stand the ball on $X$ centered in 0 of radius $r$.

2.3. Fixed points for the sum of two contractive type of mappings. The degree theory for condensing maps allows for analysis of the existence of fixed points for maps in the form of a contraction mapping plus a compact map. Recently, in [24], were given conditions under which the function results from the sum of two maps belonging to different contractive classes of mappings, satisfies a contraction inequality. With this, it is possible to establish fixed point results 
for maps resulting from the sum of two contractive types of mappings. To attain such results, a reverse triangle inequality was given in [9].

Proposition 2.9. If $\mathcal{F}: X \rightarrow \mathbb{R}$ is a linear functional of unit norm defined on the normed linear space $X$ endowed with the norm $\|\cdot\|$ and the vectors $x_{1}, \ldots, x_{n}$ satisfy the condition:

$$
0 \leq r\left\|x_{i}\right\| \leq \mathcal{F}\left(x_{i}\right), \quad i \in\{1, \ldots, n\},
$$

then

$$
r \sum_{i=1}^{n}\left\|x_{i}\right\| \leq\left\|\sum_{i=1}^{n} x_{i}\right\|,
$$

where equality holds if and only if both

$$
\mathcal{F}\left(\sum_{i=1}^{n} x_{i}\right)=r \sum_{i=1}^{n}\left\|x_{i}\right\|
$$

and

$$
\mathcal{F}\left(\sum_{i=1}^{n} x_{i}\right)=\left\|\sum_{i=1}^{n} x_{i}\right\| .
$$

In that paper (among others) mappings satisfying the next classical result were considered.

Theorem 2.10. Let $(M, d)$ be a complete metric space and $T: M \rightarrow$ $M$ a map. Then $T$ has a unique fixed point in $M$ if it satisfies any of the following conditions:

$\mathbf{B C}(\alpha)$ : (Banach, 1922, see [18]). $T$ is an $\alpha$-contraction or Banach contraction, that is,

$$
d(T x, T y) \leq \alpha d(x, y) \quad \text { for all } x, y \in M, \quad 0 \leq \alpha<1 .
$$

$\mathbf{K A}(\alpha)$ : (Kannan, 1969, 1971, $[\mathbf{1 9}, \mathbf{2 0}])$. T satisfies: there is an $\alpha \in[0,1 / 2)$ such that

$$
d(T x, T y) \leq \alpha(d(x, T x)+d(y, T y)) \quad \text { for all } x, y \in M
$$

$\mathbf{R E}\left(\mathbf{a}_{\mathbf{1}}, \mathbf{a}_{\mathbf{2}}, \mathbf{a}_{\mathbf{3}}\right):($ Reich, 1971, $[\mathbf{2 8}, \mathbf{2 9}, \mathbf{3 0}])$. T satisfies:

$$
d(T x, T y) \leq a_{1} d(x, y)+a_{2} d(x, T x)+a_{3} d(y, T y)
$$


for all $x, y \in M$ with $0 \leq a_{1}+a_{2}+a_{3}<1$.

$\mathbf{D}(\mathbf{a}, \mathbf{b}):$ (Nova, 1986, [26] or see [7, 8], for instance). $K \subset M$ is closed and $T: K \rightarrow K$ is an arbitrary operator that satisfies the following condition. For $a, b \geq 0, a+2 b<1$, and any $x, y \in K$ :

$$
d(T x, T y) \leq a d(x, y)+b[d(x, T x)+d(y, T y)] .
$$

We say that $T$ belongs or is of class $B C(\alpha)$ (respectively, $K A(\alpha)$, $R E\left(a_{1}, a_{2}, a_{3}\right)$ and $\left.D(a, b)\right)$ when $T$ satisfies the condition $B C(\alpha)$ (respectively, $K A(\alpha), R E\left(a_{1}, a_{2}, a_{3}\right)$ and $\left.D(a, b)\right)$ where $\alpha$ indicates the contraction's constant (the same indicate the parameters in each of the remainder classes).

The conditions above are, in general, independent of each other. Examples showing that can be found in [25]. Also, a comparison of these and other classes of the contractive type of mappings is given in [31].

Let $(X,\|\cdot\|)$ be a Banach space and $T, S: X \rightarrow X$ two mappings. To establish the results mentioned in [24], it was assumed that Proposition 2.9 is satisfied for $(I-T) x$ and $(I-S) x$, for each $x \in X$ which is not a fixed point of $T$ and for each $x$ which is not a fixed point of $S$; i.e.,

$$
\left\{\begin{array}{l}
0<r\|x-T x\| \leq \mathcal{F}(x-T x) \\
0<r\|x-S x\| \leq \mathcal{F}(x-S x),
\end{array} \quad \text { for all } x \in X \backslash\{x=T x \wedge x=S x\} .\right.
$$

Notice that the case when $0=r=\mathcal{F}(T x-x)=\mathcal{F}(S x-x)$ corresponds to the case when $x$ in the common fixed point for the pair $(T, S)$, which is unique or does not exist.

Theorem 2.11. Let $X$ be a Banach space, and $T, S: B_{X}(r) \rightarrow B_{X}(r)$. Assume the following conditions hold:

(a) $T \in B C(\alpha)$.

(b) For each $x \in X$, the condition (2.3) holds for $r>0$.

\section{Then:}

(i) $T+S \in D(\alpha, \beta / r)$, if $S \in K A(\beta)$.

(ii) $T+S \in R E\left(a, a_{2} / r, a_{3} / r\right)$, if $S \in R E\left(a_{1}, a_{2}, a_{3}\right), a=\alpha+a_{1}$. 
3. The nonlinear Volterra integral equation and related operators. In this paper, we are going to analyze the existence of solutions for the following class of nonlinear Volterra integral equations:

$$
u(s)=\varphi(s, u(s))+\mu \int_{a}^{b} \psi(s, t, u(t)) \mathrm{d} t, \quad s \in(a, b), \mu \in \mathbb{R}
$$

on $L^{p(\cdot)}(a, b),\left(1 \leq p_{-} \leq p(x) \leq p_{+}<\infty\right)$ with $\varphi:(a, b) \times \mathbb{R} \rightarrow \mathbb{R}$ and $\psi:(a, b) \times(a, b) \times \mathbb{R} \rightarrow \mathbb{R}$ as Carathéodory functions. That is, the functions $\varphi$ and $\psi$ satisfy the following properties:

- for every $x \in \mathbb{R}$, the function $\varphi(\cdot, x):(a, b) \rightarrow \mathbb{R}$ is Lebesgue measurable;

- for almost every $s \in(a, b)$, the function $\varphi(s, \cdot): \mathbb{R} \rightarrow \mathbb{R}$ is continuous;

- for every $x \in \mathbb{R}$, the function $\psi(\cdot, \cdot, x):(a, b) \times(a, b) \rightarrow \mathbb{R}$ is Lebesgue measurable;

- for almost every $(s, t) \in(a, b) \times(a, b)$, the function $\psi(s, t, \cdot)$ : $\mathbb{R} \longrightarrow \mathbb{R}$ is continuous.

To attain our goal, in the sequel we will assume diverse growth behaviors on the functions $\varphi$ and $\psi$.

First, we rewrite this equation in the operator theory scheme by using the operators

$$
\begin{array}{rlrl}
u \longmapsto F u, & (F u)(s) & :=\varphi(s, u(s)), \quad s \in(a, b) \\
u & \longmapsto G u, & (G u)(s):=\int_{a}^{b} \psi(s, t, u(t)) \mathrm{d} t, \quad s \in(a, b) \\
u & \longmapsto T u, & T u & :=F u+\mu G u .
\end{array}
$$

3.1. On the bounded continuity of the operators $F$ and $G$. Here, by adapting the techniques used in [17] to the case of variable exponent Lebesgue spaces, we will show that the operators $F$ and $G$ defined above are bounded and continuous on the space $L^{p(\cdot)}(a, b)$. To prove that, we will assume that the following conditions are valid:

There exists a constant $b_{1} \geq 0$ and a non-negative function $f \in L^{q(\cdot)}(a, b)$ such that, for almost every $t \in(a, b)$ and all $x \in \mathbb{R}$

$$
|\varphi(t, x)| \leq f(t)+b_{1}|x|^{p(t) / q(t)}
$$


There exists a constant $b_{2} \geq 0$ and a non-negative function $g \in L^{r(\cdot, \cdot)}((a, b) \times(a, b))$, where $r(s, t)=p(t) q(s)$, such that for almost every $(s, t) \in(a, b) \times(a, b)$ and all $x \in \mathbb{R}$

$$
|\psi(s, t, x)| \leq g(s, t)+b_{2}|x|^{p(t) / q(t)} .
$$

Here $q(\cdot)$ is a measurable function such that $p(t) \leq q(t)$ for almost every $t \in(a, b)$.

Proposition 3.1. The operator $F: L^{p(\cdot)}(a, b) \rightarrow L^{p(\cdot)}(a, b)$ is well defined, bounded and continuous.

Proof. Since $\varphi(s, u)$ is a Carathéodory function, then the operator $F$ is actually the Nemytsky operator generated by $\varphi(s, u)$; hence, from Theorem 2.5, $F: L^{p(\cdot)}(a, b) \rightarrow L^{q(\cdot)}(a, b)$ is well defined. Since $p(s) \leq q(s)$ for almost every $s \in(a, b), L^{q(\cdot)}(a, b) \hookrightarrow L^{p(\cdot)}(a, b)$. Thus, we have that $F: L^{p(\cdot)}(a, b) \rightarrow L^{p(\cdot)}(a, b)$ is well defined, bounded and continuous.

Proposition 3.2. The operator $G: L^{p(\cdot)}(a, b) \longrightarrow L^{p(\cdot)}(a, b)$ is well defined, bounded and continuous.

Proof. Let $N_{\psi}$ be the Nemytsky operator generated by the Carathéodory function $\psi(s, t, x)$. Set

$$
\begin{aligned}
& \mathcal{M}_{1}:=\{u:(a, b) \longrightarrow \mathbb{R}: u \text { is Lebesgue measurable }\} \\
& \mathcal{M}_{2}:=\{v:(a, b) \times(a, b) \longrightarrow \mathbb{R}: v \text { is Lebesgue measurable }\} .
\end{aligned}
$$

It is well known that, for every $v \in \mathcal{M}_{2}$, the function $N_{\psi} v:(a, b) \times$ $(a, b) \rightarrow \mathbb{R}$ defined by

$$
\left(N_{\psi} v\right)(s, t):=\psi(s, t, v(s, t)), \quad(s, t) \in(a, b) \times(a, b)
$$

is Lebesgue measurable. We associate to each function $u \in \mathcal{M}_{1}$ the function $v_{u} \in \mathcal{M}_{2}$ defined by

$$
v_{u}(s, t):=u(t), \quad(s, t) \in(a, b) \times(a, b) .
$$

Then, the function

$$
(s, t) \in(a, b) \times(a, b) \longmapsto \psi(s, t, u(t)) \in \mathbb{R}
$$


coincides with the function $N_{\psi} v_{u}(s, t)$ which is Lebesgue measurable. From the growth condition (3.3) and the Hölder inequality, we have that every $u \in L^{p(\cdot)}(a, b)$, the function

$$
(s, t) \in(a, b) \times(a, b) \longmapsto \psi(s, t, u(t)) \in \mathbb{R}
$$

satisfies

$$
\begin{aligned}
\int_{a}^{b} \int_{a}^{b}|\psi(s, t, u(t))| \mathrm{d} t \mathrm{~d} s \leq & \int_{a}^{b} \int_{a}^{b} g(s, t) \mathrm{d} t \mathrm{~d} s \\
& +b_{2} \int_{a}^{b} \int_{a}^{b}|u(t)|^{p(t) / q(t)} \mathrm{d} t \mathrm{~d} s \\
\leq & K_{r(\cdot, \cdot)}\|g\|_{r(\cdot, \cdot)}\|1\|_{r^{\prime}(\cdot, \cdot)} \\
& +b_{2}|b-a| \int_{a}^{b}|u(t)|^{p(t) / q(t)} \mathrm{d} t .
\end{aligned}
$$

On the other hand, we are going to prove that the function $|u|^{p(\cdot) / q(\cdot)} \in$ $L^{q(\cdot)}(a, b)$. In fact, since $0<p(t) / q(t) \leq p(t) \leq p_{+}<\infty$, then from Lemma 2.3, we have

$$
\left\|u^{p / q}\right\|_{p(\cdot) /(p(\cdot) / q(\cdot))}=\left\|u^{p / q}\right\|_{q(\cdot)} \leq\|u\|_{p(\cdot)}^{(p / q)_{ \pm}}
$$

where the $+\operatorname{sign}$ occurs when $\|u\|_{p(\cdot)} \geq 1$ and the - sign occurs in the case $\|u\|_{p(\cdot)} \leq 1$. In both cases, the last inequality is finite because $u \in L^{p(\cdot)}(a, b)$; therefore, $|u|^{p(\cdot) / q(\cdot)} \in L^{q(\cdot)}(a, b)$. Now, from the Hölder inequality, we get

$$
\int_{a}^{b}|u(t)|^{p(t) / q(t)} \mathrm{d} t \leq K_{q(\cdot)}\left\|u^{p / q}\right\|_{q(\cdot)}\|1\|_{q^{\prime}(\cdot)}<\infty
$$

thus, the inequality (3.4) is finite and then the function $\psi(s, t, u(t))$ lies in $L^{1}((a, b) \times(a, b))$. Hence, by using Fubini's theorem, it follows that, for almost every $s \in(a, b)$, the function

$$
t \in(a, b) \longmapsto \psi(s, t, u(t)) \in \mathbb{R}
$$

lies in $L^{1}(a, b)$, and the function

$$
s \in(a, b) \longmapsto \int_{a}^{b} \psi(s, t, u(t)) \mathrm{d} t \in \mathbb{R}
$$

lies in $L^{1}(a, b)$. So, we have proved that $G\left(L^{p(\cdot)}(a, b)\right) \subset L^{1}(a, b)$. 
Now, we will show that $G\left(L^{p(\cdot)}(a, b)\right) \subset L^{p(\cdot)}(a, b)$.

$$
\begin{aligned}
\int_{a}^{b}|(G u)(s)|^{p(s)} \mathrm{d} s & =\int_{a}^{b}\left|\int_{a}^{b} \psi(s, t, u(t)) \mathrm{d} t\right|^{p(s)} \mathrm{d} s \\
& \leq\left.\left.\int_{a}^{b}\left|\int_{a}^{b} g(s, t) \mathrm{d} t+b_{2} \int_{a}^{b}\right| u(t)\right|^{p(t) / q(t)} \mathrm{d} t\right|^{p(s)} \mathrm{d} s \\
& \leq 2^{p_{+}}\left[\int_{a}^{b}\left|\int_{a}^{b} g(s, t) \mathrm{d} t\right|^{p(s)} \mathrm{d} s\right. \\
& \left.+\left.\left.b_{2} \int_{a}^{b}\left|\int_{a}^{b}\right| u(t)\right|^{p(t) / q(t)} \mathrm{d} t\right|^{p(s)} \mathrm{d} s\right] .
\end{aligned}
$$

Since $g \in L^{r(\cdot, \cdot)}((a, b) \times(a, b))$, then

$$
\int_{a}^{b} \int_{a}^{b} g(s, t)^{p(t) q(s)} \mathrm{d} t \mathrm{~d} s<\infty .
$$

Thus, for almost every $t \in(a, b)$, we have

$$
\int_{a}^{b} g(s, t)^{\alpha q(s)} \mathrm{d} s<\infty, \quad \alpha:=p(t) \geq 1 .
$$

This means that the function $s \in(a, b) \mapsto g(s, t) \in \mathbb{R}$ lies in $L^{\alpha q(\cdot)}(a, b)$ and, due to the fact that $L^{\alpha q(\cdot)}(a, b) \hookrightarrow L^{p(\cdot)}(a, b)$, then $g(\cdot, t) \in$ $L^{p(\cdot)}(a, b)$. Now, from Proposition 2.2 and the integral Minkowski inequality, we obtain

$$
\begin{aligned}
\int_{a}^{b}\left|\int_{a}^{b} g(s, t) \mathrm{d} t\right|^{p(s)} \mathrm{d} s & \leq\left(\left\|\int_{a}^{b} g(\cdot, t) \mathrm{d} t\right\|_{p(\cdot)}\right)^{p_{ \pm}} \\
& \leq\left(2 K_{p(\cdot)} \int_{a}^{b}\|g(\cdot, t)\|_{p(\cdot)} \mathrm{d} t\right)^{p_{ \pm}}<\infty
\end{aligned}
$$

On the other hand, since the function $u^{p(\cdot) / q(\cdot)} \in L^{q(\cdot)}(a, b)$, from the Hölder inequality, we have

$$
\left.\left.\int_{a}^{b}\left|b_{2} \int_{a}^{b}\right| u(t)\right|^{p(t) / q(t)} \mathrm{d} t\right|^{p(s)} \mathrm{d} s \leq \int_{a}^{b}\left|b_{2} K_{q(\cdot)}\left\|u^{p / q}\right\|_{q(\cdot)}\|1\|_{q^{\prime}(\cdot)}\right|^{p(s)} \mathrm{d} s .
$$

The right side of inequality (3.5) is finite, by virtue of Proposition 2.2, the fact $1 \in L^{p(\cdot)}(a, b)$ and the homogeneity of the norm $\|\cdot\|_{p(\cdot)}$. Thus, 
$G$ is well defined and bounded on $L^{p(\cdot)}(a, b)$ into $L^{p(\cdot)}(a, b)$.

We will now prove that the operator $G$ is continuous. Let $\left(u_{n}\right)_{n} \subset$ $L^{p(\cdot)}(a, b)$ be such that

$$
\lim _{n \rightarrow \infty}\left\|u_{n}-u\right\|_{p(\cdot)}=0 .
$$

We have to show that $G u_{n} \rightarrow G u$ in $L^{p(\cdot)}(a, b)$ as $n \rightarrow \infty$. To do this, it is enough to show that any subsequence $\left(u_{n_{k}}\right)_{k}$ has a subsequence, still denoted by $\left(u_{n_{k}}\right)_{k}$, such that $G u_{n_{k}} \rightarrow G u$ in $L^{p(\cdot)}(a, b)$ as $k \rightarrow \infty$. Take $\left(u_{n_{k}}\right) \subset\left(u_{n}\right)$. It follows by Proposition 2.4 that there is a subsequence of $\left(u_{n_{k}}\right)$, still denoted by $\left(u_{n_{k}}\right)$, such that

$$
u_{n_{k}} \rightarrow u \quad \text { almost everywhere in }(a, b),
$$

and there exists $h \in L^{p(\cdot)}(a, b)$ satisfying

$$
\left|u_{n_{k}}(x)\right| \leq h(x), \quad \text { for almost every } x \in(a, b) \text { for all } k \in \mathbb{N} .
$$

Since the function $\psi(s, t, \cdot): \mathbb{R} \rightarrow \mathbb{R}$ is continuous, from (3.6), we deduce that

$$
\psi\left(s, t, u_{n_{k}}(t)\right) \longrightarrow \psi(s, t, u(t)) \text { for almost every }(s, t) \in(a, b) \times(a, b) .
$$

Now, by assumption (3.3) and inequality (3.7), we have, for almost every $(s, t) \in(a, b) \times(a, b)$,

$$
\left|\psi\left(s, t, u_{n_{k}}(t)\right)\right| \leq g(s, t)+b_{2}|h(t)|^{p(t) / q(t)} .
$$

As before, we can show that for almost every $s \in(a, b)$ the function

$$
t \in(a, b) \longmapsto g(s, t)+b_{2}|h(t)|^{p(t) / q(t)} \in \mathbb{R}
$$

lies in $L^{1}(a, b)$. Consequently, using the Lebesgue dominated theorem, we obtain

$$
\int_{a}^{b} \psi\left(s, t, u_{n_{k}}(t)\right) \mathrm{d} t \longrightarrow \int_{a}^{b} \psi(s, t, u(t)) \mathrm{d} t
$$

for almost every $s \in(a, b)$. Using again (3.3) and (3.7), we derive that, for almost every $s \in(a, b)$,

$$
\left|\int_{a}^{b} \psi\left(s, t, u_{n_{k}}(t)\right) \mathrm{d} t\right|^{p(s)} \leq 2^{p_{+}}\left[\left|\int_{a}^{b} g(s, t) \mathrm{d} t\right|^{p(s)}+\left.\left.\left|b_{2} \int_{a}^{b}\right| h(t)\right|^{p(t) / q(t)} \mathrm{d} t\right|^{p(s)}\right]
$$


and, as above, we have

$$
\begin{array}{r}
\int_{a}^{b}\left|\int_{a}^{b} g(s, t) \mathrm{d} t\right|^{p(s)} \mathrm{d} s<\infty, \\
\left.\left.\int_{a}^{b}\left|b_{2} \int_{a}^{b}\right| h(t)\right|^{p(t) / q(t)} \mathrm{d} t\right|^{p(s)} \mathrm{d} s<\infty .
\end{array}
$$

Consequently, the Lebesgue dominated theorem implies that

$$
\int_{a}^{b}\left|\int_{a}^{b} \psi\left(s, t, u_{n_{k}}(t)\right) \mathrm{d} t-\int_{a}^{b} \psi(s, t, u(t)) \mathrm{d} t\right|^{p(s)} \mathrm{d} s \longrightarrow 0,
$$

that is, $\rho_{p(\cdot)}\left(G u_{n_{k}}-G u\right) \rightarrow 0$. Thus, the continuity of $G$ is proved.

3.2. On the compactness of the operator $G$. Now, we are going to show that the operator $G$ is compact under some additional assumptions on the Carathéodory function $\psi$ and the measurable function $p(\cdot)$. More precisely, we will assume that $\psi$ satisfies the following condition:

The function $\psi$ is a Hölder continuous function on the first variable, that is,

$$
\left|\psi\left(s_{1}, t, x\right)-\psi\left(s_{2}, t, x\right)\right| \leq K\left|s_{1}-s_{2}\right|^{\lambda}, \quad K, \lambda>0 .
$$

Also, we will assume a standard condition in the framework of variable exponent Lebesgue spaces, the so-called log-Hölder continuity of an exponent $p(\cdot)$, where the function $p(\cdot)$ is called locally log-Hölder continuous if there exists a constant $C>0$ such that, for all $x, y \in \Omega$, $|x-y|<1 / 2$

$$
|p(x)-p(y)| \leq \frac{C}{-\log (|x-y|)} .
$$

The class of the all (locally) log-Hölder continuous exponents $p(\cdot)$ $\left(p_{+}<\infty\right)$ is denoted by $L H_{0}(\Omega)$.

In order to prove the compactness of the operator $G$ under assumption (3.8), we will use the Kolmogorov compactness criterion in $L^{p(\cdot)}(a, b)$.

For an integrable function $f$ on $(a, b)$, we denote by $f_{h}$ the Steklov function

$$
f_{h}(t):=\frac{1}{h} \int_{t-h / 2}^{t+h / 2} f(u) \mathrm{d} u=\frac{1}{h} \int_{-h / 2}^{h / 2} f(t+v) \mathrm{d} v .
$$


Lemma 3.3 (Kolmogorov-Tulajkov compactness criterion [27]). Let $\mathcal{F}$ be a subset of $L^{p(\cdot)}(a, b)$, and let $p(\cdot) \in L H_{0}(a, b)$. The set $\mathcal{F}$ is relatively compact if and only if

$$
\lim _{h \rightarrow 0}\left\|f_{h}-f\right\|_{p(\cdot)}=0, \quad \text { uniformly for } f \in \mathcal{F} .
$$

Proposition 3.4. For $p(\cdot) \in L H_{0}(a, b)$, under the additional assumption (3.8), the operator $G: L^{p(\cdot)}(a, b) \rightarrow L^{p(\cdot)}(a, b)$ is compact.

Proof. Let $\mathcal{F}$ be a bounded subset of $L^{p(\cdot)}(a, b)$, and let $f \in G(\mathcal{F})$. From the Kolmogorov-Tulajkov compactness criterion we must show that $\lim _{h \rightarrow 0}\left\|f_{h}-f\right\|_{p(\cdot)}=0$ uniformly for $f \in G(\mathcal{F})$.

$$
\begin{aligned}
\left|f_{h}(v)-f(v)\right| & \leq \frac{1}{h} \int_{-h / 2}^{h / 2} \int_{a}^{b}|\psi(v+w, t, u(t))-\psi(v, t, u(t))| \mathrm{d} t \mathrm{~d} w \\
& \leq \frac{K}{h} \int_{-h / 2}^{h / 2} \int_{a}^{b}|v+w-v|^{\lambda} \mathrm{d} t \mathrm{~d} w \\
& =\frac{K(b-a)}{h} \int_{-h / 2}^{h / 2}|w|^{\lambda} \mathrm{d} w=\frac{K(b-a) h^{\lambda}}{2^{\lambda}(\lambda+1)} .
\end{aligned}
$$

Since the norm in $L^{p(\cdot)}(a, b)$ is order preserving, we conclude that

$$
\left\|f_{h}-f\right\|_{p(\cdot)} \leq \frac{K(b-a) h^{\lambda}}{2^{\lambda}(\lambda+1)}\|1\|_{p(\cdot)} .
$$

Therefore, the uniform convergence follows, so the operator $G$ is compact.

\section{Existence of solutions of the Volterra integral equation} (3.1). In this section, we will provide conditions under which the nonlinear integral Volterra equation (3.1) has at least one solution. First, we will use topological degree theory for condensing maps. Afterward, fixed points results for the sum of contractive type of mappings will be used.

\subsection{On the solutions of equation (3.1) by means of condensing} maps. In Section 3, we proved that, under some conditions on the Carathéodory functions $\varphi$ and $\psi$, the operators $F$ and $G$ are bounded and continuous in $L^{p(\cdot)}(a, b)$; even more, $G$ is a compact operator. This 
allows us to use topological degree theory for condensing maps and the corresponding fixed point Theorem 2.8.

Theorem 4.1. If the functions $\varphi:(a, b) \times \mathbb{R} \rightarrow \mathbb{R}$ and $\psi:(a, b) \times$ $(a, b) \times \mathbb{R} \rightarrow \mathbb{R}$ satisfy conditions (3.2) and (3.3), if the function $\varphi$ is a strict contraction on the second variable, i.e.,

$$
|\varphi(t, x)-\varphi(t, y)| \leq L|x-y|, \quad 0 \leq L<1
$$

and the function $\psi$ satisfies assumption (3.8), then the nonlinear integral equation (3.1)

$$
\begin{gathered}
u(s)=\varphi(s, u(s))+\mu \int_{a}^{b} \psi(s, t, u(t)) \mathrm{d} t, \\
s \in(a, b), \quad \mu \in \mathbb{R}
\end{gathered}
$$

has at least one solution $u \in L^{p(\cdot)}(a, b)$, for $p(\cdot) \in L H_{0}(a, b)$, satisfying $(p / q)_{+}<1$ and the set of the solutions of equation (3.1) is bounded in $L^{p(\cdot)}(a, b)$.

Proof. Let $F, G, T: L^{p(\cdot)}(a, b) \rightarrow L^{p(\cdot)}(a, b)$ be the operators defined in Section 3 which are continuous, bounded and, moreover, $G$ is compact. From Proposition 2.7 (ii), the operator $\mu G$ is $\mu \alpha$-Lipschitz with zero constant. Since the function $\varphi$ is a strictly contraction, it follows that, for every $u, v \in L^{p(\cdot)}(a, b)$,

$$
\|F u-F v\|_{p(\cdot)} \leq L\|u-v\|_{p(\cdot)},
$$

which means that $F$ is a Lipschitz map with constant $L$ and so, Proposition 2.7(iii) and (i) guarantee that $F$ is $\alpha$-Lipschitz with constant $L$, and $T$ is a strict $\alpha$-contraction with constant $L$.

Set

$$
\mathcal{G}=\left\{u \in L^{p(\cdot)}(a, b): \text { there exists } \lambda \in[0,1] \text { such that } u=\lambda T u\right\} .
$$

We will prove that the set $\mathcal{G}$ is bounded in $L^{p(\cdot)}(a, b)$. In fact, for all $u \in \mathcal{G}$

$$
\|u\|_{p(\cdot)}=\lambda\|T u\|_{p(\cdot)} \leq \lambda\left(\|F u\|_{p(\cdot)}+|\mu|\|G u\|_{p(\cdot)}\right) .
$$

First, we will estimate an upper bound for $\|F u\|_{p(\cdot)}$. To do this, we use the growth condition (3.2) and the following facts: (i) the $L^{p(\cdot)}$-norm is order preserving, (ii) the triangle inequality, (iii) $L^{q(\cdot)}(\Omega) \hookrightarrow L^{p(\cdot)}(\Omega)$, 
which implies that there exists $K>1$ such that, for all $f \in L^{q(\cdot)}(a, b)$, $\|f\|_{p(\cdot)} \leq K\|f\|_{q(\cdot)}$ and (iv) Lemma 2.3.

$$
\begin{aligned}
\|F u\|_{p(\cdot)} & \leq\left\|f+b_{1}|u|^{p / q}\right\|_{p(\cdot)} \\
& \leq\|f\|_{p(\cdot)}+b_{1}\left\|u^{p / q}\right\|_{p(\cdot)} \\
& \leq K\|f\|_{q(\cdot)}+b_{1} K\left\|u^{p / q}\right\|_{q(\cdot)} \\
& \leq K\|f\|_{q(\cdot)}+b_{1} K\|u\|_{p(\cdot)}^{(p / q)_{ \pm}}
\end{aligned}
$$

Now, we will give an upper bound for $\|G u\|_{p(\cdot)}$. In this case, we use the growth condition (3.3) and the following facts: (i) the $L^{p(\cdot)}$-norm is order preserving, (ii) the triangle inequality, (iii) the Hölder inequality, (iv) the Minkoswski inequality and (v) Lemma 2.3.

$$
\begin{aligned}
\|G u\|_{p(\cdot)} & \leq\left\|\int_{a}^{b} g(\cdot, t) \mathrm{d} t\right\|_{p(\cdot)}+\left\|\int_{a}^{b} b_{2}|u(t)|^{p(t) / q(t)} \mathrm{d} t\right\|_{p(\cdot)} \\
& \leq\left\|\int_{a}^{b} g(\cdot, t) \mathrm{d} t\right\|_{p(\cdot)}+b_{2} \int_{a}^{b}|u(t)|^{p(t) / q(t)} \mathrm{d} t\|1\|_{p(\cdot)} \\
& \leq 2 K_{p(\cdot)} \int_{a}^{b}\|g(\cdot, t)\|_{p(\cdot)} \mathrm{d} t+b_{2} K_{q(\cdot)}\|u\|_{p(\cdot)}^{(p / q)_{ \pm}}\|1\|_{q^{\prime}(\cdot)}\|1\|_{p(\cdot)} .
\end{aligned}
$$

Thus, with these upper bounds, we obtain the following estimate for all $u \in \mathcal{G}$,

$$
\begin{aligned}
\|u\|_{p(\cdot)} \leq & \lambda\left(\|F u\|_{p(\cdot)}+|\mu|\|G u\|_{p(\cdot)}\right) \\
\leq & \lambda\left(K\|f\|_{q(\cdot)}+b_{1} K\|u\|_{p(\cdot)}^{(p / q)_{ \pm}}\right) \\
& +\lambda|\mu|\left(2 K_{p(\cdot)} \int_{a}^{b}\|g(\cdot, t)\|_{p(\cdot)} \mathrm{d} t\right. \\
& \left.+b_{2} K_{q(\cdot)}\|u\|_{p(\cdot)}^{(p / q)_{ \pm}}\|1\|_{q^{\prime}(\cdot)}\|1\|_{p(\cdot)}\right) .
\end{aligned}
$$

Notice that the fact $0<(p / q)_{ \pm}<1$, together with the inequality above, shows that $\mathcal{G}$ is bounded in $L^{p(\cdot)}(a, b)$. Consequently, by Theorem 2.8, the operator $T$ has at least a fixed point, and the set of the fixed points of $T$ is bounded in $L^{p(\cdot)}(a, b)$. Therefore, the nonlinear integral equation (3.1) has at least one solution $u \in L^{p(\cdot)}(a, b)$, and the set of the solutions of the equation is bounded in $L^{p(\cdot)}(a, b)$. 
4.2. Existence and uniqueness of solutions of equation (3.1) through the sum of contractive type of mappings. As we saw, if the function $\varphi$ is $\alpha$-contractive in the second variable, then the operator $F$ is a Banach contraction mapping in $L^{p(\cdot)}(a, b)$ with constant $\alpha$. Now, we will prove that, if $\psi$ is a Lipschitz function, then the induced operator $G$ is a Lipschitz operator in $L^{p(\cdot)}(a, b)$. Then, the existence and uniqueness of the solutions of equation (3.1) in this case will be a consequence of the fact that the sum of two Banach contraction mappings is a Banach contraction mapping.

Proposition 4.2. If the function $\psi$ satisfies (3.3) and is Lipschitz on the third variable with constant $L>0$, i.e.,

$$
|\psi(s, t, x)-\psi(s, t, y)| \leq L|x-y|, \quad \text { for all } x, y \in(a, b), L>0
$$

then the induced operator $G$ is Lipschitz on the space $L^{p(\cdot)}(a, b)$ with constant $\lambda=L K_{p(\cdot)}\|1\|_{p(\cdot)}\|1\|_{p^{\prime}(\cdot)}$.

Proof. Let $u, v \in L^{p(\cdot)}(a, b)$. From Proposition 3.2, the operator $G$ is bounded and continuous on $L^{p(\cdot)}(a, b)$ (but not necessarily compact because the condition $p(\cdot) \in L H_{0}(a, b)$ is not required). Thus, using the Hölder inequality, we obtain

$$
\begin{aligned}
\|G u-G v\|_{p(\cdot)} & =\left\|\int_{a}^{b}[\psi(s, t, u(t))-\psi(s, t, v(t))] \mathrm{d} t\right\|_{p(\cdot)} \\
& \leq\left\|L \int_{a}^{b}|u(t)-v(t)| \mathrm{d} t\right\|_{p(\cdot)} \\
& \leq L \int_{a}^{b}|u(t)-v(t)| \mathrm{d} t \cdot\|1\|_{p(\cdot)} \\
& \leq L K_{p(\cdot)}\|u-v\|_{p(\cdot)}\|1\|_{p^{\prime}(\cdot)}\|1\|_{p(\cdot)},
\end{aligned}
$$

that is, $G$ is Lipschitz on $L^{p(\cdot)}(a, b)$ with constant

$$
\lambda=L K_{p(\cdot)}\|1\|_{p(\cdot)}\|1\|_{p^{\prime}(\cdot)} .
$$

This completes the proof. 
Theorem 4.3. If the function $\varphi:(a, b) \times \mathbb{R} \rightarrow \mathbb{R}$ satisfies (3.2) and is a strict contraction on the second variable, i.e.,

$$
|\varphi(t, x)-\varphi(t, y)| \leq M|x-y|
$$

for $M<1$, and $\psi:(a, b) \times(a, b) \times \mathbb{R} \rightarrow \mathbb{R}$ satisfies conditions (3.3) and (4.1), with $L>0$ sufficiently small, then the nonlinear integral equation $(3.1)$ :

$$
u(s)=\varphi(s, u(s))+\mu \int_{a}^{b} \psi(s, t, u(t)) \mathrm{d} t, \quad s \in(a, b), \mu \in \mathbb{R}
$$

has a unique solution $u \in L^{p(\cdot)}(a, b)$.

Proof. From equation (3.2) and the fact that $\varphi$ is a strict contraction on the second variable with constant $M$, the operator $F$ is a Banach contraction on $L^{p(\cdot)}(a, b)$ with constant $M$. Conditions (3.3) and (4.1) assure that the operator $G$ is a Lipschitz operator on $L^{p(\cdot)}(a, b)$ with constant $\lambda=L K_{p(\cdot)}\|1\|_{p(\cdot)}\|1\|_{p^{\prime}(\cdot)}$. Therefore, $T=F+\mu G$ is a Lipschitz operator with constant

$$
\kappa=M+L|\mu| K_{p(\cdot)}\|1\|_{p(\cdot)}\|1\|_{p^{\prime}(\cdot)} .
$$

Choosing $L$ such that $\kappa<1$, then $T$ is Banach contraction operator on $L^{p(\cdot)}(a, b)$ and therefore has a unique fixed point. Thus, equation (3.1) has a unique solution on $L^{p(\cdot)}(a, b)$.

Now, let us impose some, less restrictive, contraction's conditions on the Carathéodory function $\varphi$, such that the induced operator $F$ is a contractive type of mapping. In this case, the existence and uniqueness of the solutions of equation (3.1) will be a consequence of the existence of a unique fixed point for the mapping result from sum of two contractive type of mappings.

Proposition 4.4. If the function $\varphi$ satisfies that

$$
|\varphi(s, u(s))| \leq \frac{\lambda}{\lambda+1}|u(s)|, \quad \lambda \in[0,1 / 2), \quad u \in L^{p(\cdot)}(a, b),
$$

then the operator $F$ is a Kannan contraction operator on $L^{p(\cdot)}(a, b)$.

Proof. First notice that the operator $F$ is bounded on $L^{p(\cdot)}(a, b)$ if we replace $(3.2)$ by $(4.2)$. Let $u, v \in L^{p(\cdot)}(a, b)$. From the triangle 
inequality and (4.2) we have that

$$
\begin{aligned}
|\varphi(s, u(s))-\varphi(s, v(s))| & \leq|\varphi(s, u(s))|+|\varphi(s, v(s))| \\
& \leq \lambda|u(s)|-\lambda \varphi|(s, u(s))|+\lambda|v(s)|-|\varphi(s, v(s))| \\
& \leq \lambda[|u(s)-\varphi(s, u(s))|+|v(s)-\varphi(s, v(s))|] .
\end{aligned}
$$

Therefore, taking the $L^{p(\cdot)}$-norm, we have

$$
\begin{gathered}
\|F u-F v\|_{p(\cdot)} \leq \lambda\left[\|u-F u\|_{p(\cdot)}+\|v-F v\|_{p(\cdot)}\right] \\
0 \leq \lambda<1 / 2 .
\end{gathered}
$$

Thus, $F$ is a Kannan contraction on $L^{p(\cdot)}(a, b)$ with constant $\lambda$.

Proposition 4.5. If the function $\varphi$ satisfies that

$$
|\varphi(s, u(s))| \leq \frac{\lambda}{\lambda+1}|u(s)|, \quad \lambda \in[0,1 / 3), u \in L^{p(\cdot)}(a, b),
$$

then the operator $F$ is a Reich contraction operator on $L^{p(\cdot)}(a, b)$.

Proof. Let $u, v \in L^{p(\cdot)}(a, b)$. As in the proof of proposition above, notice that the operator $F$ is bounded on $L^{p(\cdot)}(a, b)$ if we replace (3.2) by (4.3). In addition, from the triangle inequality, we have that

$$
\begin{aligned}
|\varphi(s, u(s))-\varphi(s, v(s))| \leq & \lambda|u(s)-\varphi(s, u(s))|+\lambda|v(s)-\varphi(s, v(s))| \\
\leq & \lambda^{*}|u(s)-v(s)|+\lambda|u(s)-\varphi(s, u(s))| \\
& +\lambda|v(s)-\varphi(s, v(s))|, \quad \text { for } 0 \leq \lambda^{*}<1 / 3 .
\end{aligned}
$$

Therefore, taking the $L^{p(\cdot)}$-norm, we have

$$
\begin{aligned}
\|F u-F v\|_{p(\cdot)} \leq & \lambda^{*}\|u-v\|_{p(\cdot)}+\lambda\|u-F u\|_{p(\cdot)} \\
& +\lambda\|v-F v\|_{p(\cdot)}
\end{aligned}
$$

with $\lambda^{*}, \lambda \in[0,1 / 3)$. Thus, $F$ is a Reich contraction on $L^{p(\cdot)}(a, b)$.

By $B_{p(\cdot)}(r)$, we set the ball centered on 0 with radius $r$ on the space $L^{p(\cdot)}(a, b)$. Let, $\mathcal{F}$ be a linear functional of unit norm on $L^{p(\cdot)}(a, b)$ such that

$$
\begin{cases}0<r\|u-\mu G u\|_{p(\cdot)} \leq \mathcal{F}(u-\mu G u) & \text { for all } u \in B_{p(\cdot)}(r) \backslash\{u=\mu G u\}, \\ 0<r\|u-F u\|_{p(\cdot)} \leq \mathcal{F}(u-F u) & \text { for all } u \in B_{p(\cdot)}(r) \backslash\{u=F u\} .\end{cases}
$$


Notice that the operator $F$ adds $B_{p(\cdot)}(r)$ into $B_{p(\cdot)} 1 /(1+\lambda) r$ if the function $\varphi$ satisfies (4.2) or (4.3). For the operator $\mu G$, the following result holds.

Lemma 4.6. For $|\mu|$ sufficiently small, the operator $\mu G$ adds $B_{p(\cdot)}(r)$ into $B_{p(\cdot)} 1 /(1+\lambda) r$.

Proof. Let $u \in B_{p(\cdot)}(r)$. Then, from the proof of Proposition 3.2, by Proposition 2.2 and the Hölder inequality, we have the following estimation:

$$
\begin{aligned}
\|G u\|_{p(\cdot)}^{p_{\mp} \leq} & 2^{p_{+}}\left(2 K_{p(\cdot)} \int_{a}^{b}\|g(\cdot, t)\|_{p(\cdot)} \mathrm{d} t\right)^{p_{ \pm}} \\
& +2^{p_{+}} \int_{a}^{b}\left|b_{2} K_{q(\cdot)}\|u\|_{p(\cdot)}^{(p / q)_{ \pm}}\|1\|_{q^{\prime}(\cdot)}\right|^{p(s)} \mathrm{d} s \\
\leq & 2^{p_{+}}\left(2 K_{p(\cdot)} \int_{a}^{b}\|g(\cdot, t)\|_{p(\cdot)} \mathrm{d} t\right)^{p_{ \pm}} \\
& +2^{p_{+}}\left\|b_{2} K_{q(\cdot)}\right\| u\left\|_{p(\cdot)}^{(p / q)_{ \pm}}\right\| 1\left\|_{q^{\prime}(\cdot)}\right\|_{p_{ \pm}(\cdot)}^{p_{ \pm}} \\
\leq & 2^{p_{+}}\left(2 K_{p(\cdot)} \int_{a}^{b}\|g(\cdot, t)\|_{p(\cdot)} \mathrm{d} t\right)^{p_{ \pm}} \\
& +2^{p_{+}}\left(r^{(p / q)_{ \pm}} b_{2} K_{q(\cdot)}\|1\|_{q^{\prime}(\cdot)}\|1\|_{p(\cdot)}\right)^{p_{ \pm}}
\end{aligned}
$$

Therefore,

$$
\begin{aligned}
& \|\mu G u\|_{p(\cdot)}^{p_{\mp}} \leq|\mu|^{p_{\mp}} 2^{p_{+}}\left[\left(2 K_{p(\cdot)}\|\| g(\cdot, t)\left\|_{p(\cdot)}\right\|_{1}\right)^{p_{ \pm}}\right. \\
& \left.+\left(\left(\frac{1}{1+\lambda} r\right)^{p_{\mp}} b_{2} K_{q(\cdot)}\|1\|_{q^{\prime}(\cdot)}\|1\|_{p(\cdot)}\right)^{p_{ \pm}}\right] .
\end{aligned}
$$

By choosing $|\mu|$ sufficiently small such that the right side of the inequality above is less than $r$, the conclusion follows.

The next result proves the existence of a unique solution of equation (3.1), in the case when the behavior of the function $\varphi$ is such that the induced operator $F$ is a Kannan or Reich mapping.

Theorem 4.7. If the function $\varphi:(a, b) \times \mathbb{R} \longrightarrow \mathbb{R}$ satisfies (4.2) or (4.3) and $\psi:(a, b) \times(a, b) \times \mathbb{R} \rightarrow \mathbb{R}$ satisfies conditions (3.3) and (4.1), 
then the nonlinear integral equation (3.1)

$$
u(s)=\varphi(s, u(s))+\mu \int_{a}^{b} \psi(s, t, u(t)) \mathrm{d} t, \quad s \in(a, b),|\mu| \ll 1,
$$

has a unique solution $u \in B_{p(\cdot)}(r)$, for a convenient choice of the parameters $\mu, \lambda$ and $r$.

Proof. Let $T=F+\mu G: B_{p(\cdot)}(r) \rightarrow B_{p(\cdot)}(r)$ be the associated operator to the equation (3.1). For $|\mu|$ sufficiently small, $T$ acts over functions $u \in B_{p(\cdot)}(r)$ satisfying the condition $(2.3)$ in $B_{p(\cdot)}(r)$.

If the function $\varphi$ satisfies (4.2), by Proposition 4.4, $F \in K A(\lambda)$ and if $\varphi$ satisfies (4.3), from Proposition 4.5, $F \in R E\left(\lambda^{*}, \lambda, \lambda\right)$. On the other hand, Proposition 4.2 gives that $\mu G \in B C\left(|\mu| \lambda_{L}\right)$, with $\lambda_{L}=L K_{p(\cdot)}\|1\|_{p(\cdot)}\|1\|_{p^{\prime}(\cdot)}$.

Thus, from Theorem 2.11, we conclude that $T$ belongs to one of the following classes:

- $T \in D\left(|\mu| \lambda_{L}, \lambda / r\right)$.

- $T \in R E\left(\lambda^{*}+|\mu| \lambda_{L}, \lambda / r, \lambda / r\right)$.

In both cases, by virtue of Theorem 2.10, the operator $T$ has a unique fixed point on $B_{p(\cdot)}(r)$ for a convenient choice of the contractive's parameters. Therefore, equation (3.1) has a unique solution $u \in$ $B_{p(\cdot)}(r)$.

4.3. Continuous solutions of equation (3.1). Since, in the case $p_{+}<\infty$, the set $C(a, b) \cap L^{p(\cdot)}(a, b)$ as well as the set of continuous functions with finite support are dense in $L^{p(\cdot)}(a, b)$, this section is devoted to studying the existence of continuous solutions of equation (3.1). To attain this aim, we are going to use Schaefer's fixed point theorem and Arzelà-Ascoli's theorem. For the sake of the presentation we enunciate both results here.

Theorem 4.8 (Arzelà-Ascoli). A necessary and sufficient condition for a family of continuous functions defined on the compact interval $[a, b]$ to be compact in $C[a, b]$ is that this family is uniformly bounded and equicontinuous. 
Theorem 4.9 (Schaefer's fixed point theorem). Assume that $X$ is a Banach space and that $T: X \rightarrow X$ is a continuous compact mapping. Moreover, assume that the set

$$
\bigcup_{0 \leq \lambda \leq 1}\{x \in X: x=\lambda T x\}
$$

is bounded. Then $T$ has a fixed point.

The existence of continuous solutions of equation (3.1) is established in the following result.

Theorem 4.10. If the function $\varphi:[a, b] \times \mathbb{R} \rightarrow \mathbb{R}$ satisfies (3.2) with $f \in L^{\infty}(a, b)$ and is continuous, and if the function $\psi:[a, b] \times[a, b] \times$ $\mathbb{R} \rightarrow \mathbb{R}$ satisfies (3.3) with $M:=\sup _{s \in[a, b]}\left|\int_{a}^{b} g(s, t) \mathrm{d} t\right|<\infty$ and is continuous, then the nonlinear integral equation (3.1):

$$
u(s)=\varphi(s, u(s))+\mu \int_{a}^{b} \psi(s, t, u(t)) \mathrm{d} t, \quad s \in(a, b), \mu \in \mathbb{R}
$$

has a solution $u \in C[a, b]$.

Proof. We prove the existence of continuous solutions of equation (3.1) by establishing the existence of fixed points of the associated operator $T=F+\mu G$. To attain such a goal, we are going to apply Theorem 4.9; therefore, we need to show that the operator $T$ applies $C[a, b]$ into itself, is continuous and compact.

First, we will prove that $T(C[a, b]) \subset C[a, b]$. Let us consider an arbitrary function $u \in C[a, b]$, and let $s, s_{0} \in[a, b]$. Then, we get

$$
\begin{aligned}
\left|(T u)(s)-(T u)\left(s_{0}\right)\right| \leq & \left|\varphi(s, u(s))-\varphi\left(s_{0}, u\left(s_{0}\right)\right)\right| \\
& +|\mu| \int_{a}^{b}\left|\psi(s, t, u(t))-\psi\left(s_{0}, t, u(t)\right)\right| \mathrm{d} t .
\end{aligned}
$$

Since there exists $M>0$ such that $|u(t)| \leq M$, for all $t \in[a, b]$, then $u$ is uniformly continuous. This implies that the function $\varphi$ : $[a, b] \times[-M, M] \rightarrow \mathbb{R}$ is uniformly continuous; that is, for each $\epsilon / 2>0$, there exists $\delta_{1}>0$ such that

$$
\left|\varphi(s, u(s))-\varphi\left(s_{0}, u\left(s_{0}\right)\right)\right|<\frac{\epsilon}{2}
$$


for all $(s, u(s)),\left(s_{0}, u\left(s_{0}\right)\right) \in[a, b] \times[-M, M]$, satisfying that $0<$ $\left\|(s, u(s))-\left(s_{0}, u\left(s_{0}\right)\right)\right\|_{\infty}=\max \left\{\left|s-s_{0}\right|,\left|u(s)-u\left(s_{0}\right)\right|\right\}<\delta_{1}$.

Similarly, for each $\epsilon /[2(|\mu|+1)(b-a)]>0$, there exists $\delta_{2}>0$ such that

$$
\left|\psi(s, t, u(t))-\psi\left(s_{0}, t, u(t)\right)\right|<\frac{\epsilon}{2(|\mu|+1)(b-a)}
$$

for all $(s, t, u(t)),\left(s_{0}, t, u(t)\right) \in[a, b] \times[a, b] \times[-M, M]$ satisfying $0<\left\|(s, t, u(t))-\left(s_{0}, t, u(t)\right)\right\|_{\infty}=\left|s-s_{0}\right|<\delta_{2}$.

Taking $\delta=\min \left\{\delta_{1}, \delta_{2}\right\}$, from inequality (4.4), we conclude that

$$
\begin{aligned}
\left|(T u)(s)-(T u)\left(s_{0}\right)\right| & <\frac{\epsilon}{2}+|\mu| \int_{a}^{b} \frac{\epsilon}{2(|\mu|+1)(b-a)} \mathrm{d} t \\
& =\frac{\epsilon}{2}+\frac{|\mu|}{1+|\mu|} \frac{\epsilon}{2} \leq \epsilon .
\end{aligned}
$$

Therefore, $(T u)(s)$ is a (uniformly) continuous function, for any $u \in$ $C[a, b]$. Hence, $T(C[a, b]) \subset C[a, b]$. Actually, since the previous conclusion does not depend on the function $u$, we prove in fact, that the set $T(C[a, b]) \subset C[a, b]$ is equicontinuous.

The continuity of $T$ on $C[a, b]$ follows from the continuity of $T$ on $L^{p(\cdot)}[a, b]$ and the fact that $C[a, b] \subset L^{p(\cdot)}[a, b]$. Now, we are going to prove the compactness of $T$. It suffices to check that the conditions of Arzelà-Ascoli's theorem are satisfied for $\overline{T\left(B_{\infty}(1)\right)}$, that is, the closure on $C[a, b]$ of the image under $T$ of the closed unit ball $B_{\infty}(1)$. Since $T$ is a continuous operator, the set $T\left(B_{\infty}(1)\right)$ is bounded. The equicontinuity of $\overline{T\left(B_{\infty}(1)\right)}$ follows from the equicontinuity of $T(C[a, b])$. Therefore, the operator $T: C[a, b] \rightarrow C[a, b]$ is compact.

Finally, we are going to prove that for each $\lambda \in[0,1]$ the sets

$$
\mathcal{G}_{\lambda}:=\{u \in C[a, b]: u=\lambda T u\}
$$

are bounded. In fact, from conditions (3.2) and (3.3), for $u \in \mathcal{G}_{\lambda}$, we get

$$
\begin{aligned}
\|u\|_{\infty} & =\lambda\|T u\|_{\infty} \leq \lambda\|\varphi(s, u(s))\|_{\infty}+\lambda\left\|\mu \int_{a}^{b} \psi(s, t, u(t)) \mathrm{d} t\right\|_{\infty} \\
& \leq \lambda\left(\|f\|_{\infty}+b_{1}\left\|u^{p / q}\right\|_{\infty}+|\mu|\left\|\int_{a}^{b}\left(g(s, t)+b_{2}|u(t)|^{p(t) / q(t)}\right) \mathrm{d} t\right\|_{\infty}\right)
\end{aligned}
$$




$$
\begin{aligned}
\leq & \lambda\left(\|f\|_{\infty}+b_{1}\left\|u^{p / q}\right\|_{\infty}\right) \\
& +\lambda|\mu| \sup _{s \in[a, b]}\left\{b_{2}(b-a)\left\|u^{p / q}\right\|_{\infty}+\int_{a}^{b} g(s, t) \mathrm{d} t\right\} \\
= & \lambda\left(\|f\|_{\infty}+\left(b_{1}+b_{2}(b-a)|\mu|\right)\left\|u^{p / q}\right\|_{\infty}+|\mu| M\right) .
\end{aligned}
$$

Notice that, from the fact that $1 \in L^{p(\cdot)}[a, b]$ for any function $p(\cdot)$ with $p_{+}<\infty,\left[4\right.$, Proposition 2.43] guarantees that $L^{\infty}[a, b] \subset L^{p(\cdot)}[a, b]$, so for all $f \in L^{\infty}[a, b],\|f\|_{p(\cdot)} \leq\|1\|_{p(\cdot)}\|f\|_{\infty}$. Therefore, for all functions $f \in C[a, b] \subset L^{\infty}[a, b]$, we can find a constant $K>0$ such that $\|f\|_{\infty} \leq K\|f\|_{q(\cdot)}$. Using this fact and Lemma 2.3, we have

$$
\begin{aligned}
\left\|u^{p / q}\right\|_{\infty} & \leq K\left\|u^{p / q}\right\|_{q(\cdot)} \leq K\|u\|_{p(\cdot)}^{(p / q)_{ \pm}} \\
& \leq K\|1\|_{p(\cdot)}^{(p / q)_{ \pm}}\|u\|_{\infty}^{(p / q)_{ \pm}}
\end{aligned}
$$

Therefore, from inequality (4.5), we have

$$
\begin{aligned}
\|u\|_{\infty} & =\lambda\|T u\|_{\infty} \\
& \leq \lambda\left(\|f\|_{\infty}+\left(b_{1}+b_{2}(b-a)|\mu|\right) K\|1\|_{p(\cdot)}^{(p / q)_{ \pm}}+|\mu| M\right) .
\end{aligned}
$$

Hence, each set $\mathcal{G}_{\lambda}$ is bounded, and so $\cup_{0 \leq \lambda \leq 1} \mathcal{G}_{\lambda}$. Applying Schaefer's fixed point theorem, the operator $T$ has a fixed point in $C[a, b]$, or equivalently, equation (3.1) has a solution on $C[a, b]$. The proof is complete.

Remark 4.11. Notice that the growth conditions (3.2) and (3.3), imposed on the functions $\varphi$ and $\psi$, respectively, are related to the exponent functions on the underlying spaces $L^{p(\cdot)}$. Theorem 4.10 establishes conditions for the existence of solutions on $C[a, b]$ of the equation (3.1) and, as we saw, the proof takes into consideration the relations between the norm of $C[a, b]$ and the norm of $L^{p(\cdot)}[a, b]$ (see, inequalities (4.6)).

Since the main goal of this paper is to analyze the existence of solutions of the equation (3.1) defined on $L^{p(\cdot)}(a, b)$, Theorem 4.10 becomes a tool to guarantee the existence of continuous solutions of equation (3.1) in this framework, and takes more relevance from the fact that the set of continuous functions whose support is compact and contained in $(a, b)$ is dense in $L^{p(\cdot)}(a, b)$ (upon, $p_{+}<\infty$ ). 
The existence of solutions of equation (3.1) on $C[a, b]$, in the case when the growth conditions (3.2) and (3.3) of the Carathéodory functions $\varphi$ and $\psi$ are not related to the norm of the space discussed in [16].

The uniqueness of the continuous solutions of the integral equation (3.1) is given in the following result.

Theorem 4.12. If the function $\varphi:[a, b] \times \mathbb{R} \rightarrow \mathbb{R}$ is continuous and is a strict contraction in the second variable, i.e.,

$$
|\varphi(t, x)-\varphi(t, y)| \leq L|x-y|, \quad 0 \leq L<1
$$

and if the function $\psi:[a, b] \times[a, b] \times \mathbb{R} \rightarrow \mathbb{R}$ is continuous and is Lipschitz on the third variable, i.e.,

$$
|\psi(t, s, x)-\varphi(t, s, y)| \leq M|x-y|, \quad M \geq 0,
$$

then the nonlinear integral equation (3.1):

$$
u(s)=\varphi(s, u(s))+\mu \int_{a}^{b} \psi(s, t, u(t)) \mathrm{d} t, \quad s \in(a, b), \mu \in \mathbb{R}
$$

has a unique solution $u \in C[a, b]$ whenever $L+|\mu| M(b-a)<1$.

Proof. Let $u, v \in C[a, b]$. Since the Carathéodory functions $\varphi$ and $\psi$ are continuous, in Theorem 4.10, it was proved that $T(C[a, b]) \subset$ $C[a, b]$. Now, from the triangle inequality and the Lipschitz conditions on the functions $\varphi$ and $\psi$ we get

$$
\begin{aligned}
\|T u-T v\|_{\infty} \leq & \|\varphi(s, u)-\varphi(s, v)\|_{\infty} \\
& +\left\|\mu \int_{a}^{b}[\psi(s, t, u(t))-\psi(s, t, v(t))] \mathrm{d} t\right\|_{\infty} \\
\leq & (L+|\mu|(b-a) M)\|u-v\|_{\infty} .
\end{aligned}
$$

That means the operator $T: C[a, b] \rightarrow C[a, b]$ belongs to $B C(\alpha)$ with $\alpha=(L+|\mu|(b-a) M)<1$. Thus, from Theorem 2.10, $T$ has a unique fixed point, obtaining in this way the conclusion.

Remark 4.13. Notice that Theorem 4.3 guarantees the existence of a unique solution of equation (3.1) on the whole space $L^{p(\cdot)}(a, b)$, whereas 
Theorem 4.12 guarantees the existence of a unique continuous solution of this equation. Thus, as Theorem 4.10, under the assumptions (3.2) and (3.3), it can be useful to extract the continuous solutions of equation (3.1).

Final remarks. We would like to point out that the use of fixed point theorems for the mappings resulting from the sum of two contractive-type maps (Theorem 2.11) don't require continuity or compactness of the associate operators. Consequently, via this result, we can analyze the existence of solutions of Volterra integral type equations with kernels having a diverse kind of growth behavior, without imposing continuity or compactness conditions to the operators involved. It also can be performed on different function spaces. To the best of our knowledge, it has not been considered yet.

Acknowledgments. The authors are grateful to the referee whose comments and suggestions led to an improvement of several proofs in the paper.

\section{REFERENCES}

1. H. Brunner, Collocation methods for Volterra integral and related functional differential equations, Cambr. Mono. Appl. Comp. Math. 15, Cambridge University Press, Cambridge, 2004.

2. T.A. Burton, Volterra integral and differential equations, 2nd edition, Math. Sci. Engin. 202, Elseiver, Amsterdam, 2005.

3. C. Corduneanu, Principles of differential and integral equations, 2nd edition, Chelsea, New York, 1988.

4. D.V. Cruz-Uribe and A. Fiorenza, Variable Lebesgue spaces, Foundations and harmonic analysis, Appl. Num. Harm. Anal., Birkhäuser, Basel, 2013.

5. D.V. Cruz-Uribe, A. Fiorenza and M.V. Ruzhansky, Variable Lebesgue Spaces and hyperbolic systems, Adv. Courses Math.-CRM Barcelona, Birkhäuser, 2014.

6. K. Deimling, Nonlinear functional analysis, Springer-Verlag, 1985.

7. W.R. Derryck and L. Nova, Interior properties and fixed points of certain discontinuous operators, Elsevier Science, 1992, 239-245.

8. (1989), 339-347.

Fixed point theorems for discontinuous operators, Glasnik Matem.

9. J.B. Diaz and F.T. Metcalf, A complementary triangle inequality in Hilbert and Banach spaces, Proc. Amer. Math. Soc. 17 (1966), 88-97.

10. L. Diening, P. Harjulehto, P. Hästö and M. Růžička, Lebesgue and Sobolev spaces with variable exponents, Lect. Notes Math. 2017, Springer, 2011. 
11. G. Dinca and F. Isaia, Generalized Pohožaev and Pucci-Serrin identities and non-existence results for $p(x)$-Laplacian type equations, Rend. Circ. Matem. Palermo 59 (2010), 1-46.

12. D.E. Edmunds and W.D. Evans, Hardy operators, function spaces and embeddings, Springer-Verlag, Berlin, 2004.

13. X. Fan and D. Zhao, On the spaces $L^{p(\cdot)}(\Omega)$ and $W^{m, p(\cdot)}(\Omega)$, J. Math. Anal. Appl. 263 (2001), 424-446.

14. A. Gogatishvili and J. Lang, Generalized Hardy operators with kernel and variable integral limits in Banach function spaces, J. Inequal. Appl. 4 (1999), 1-16.

15. G. Gripenberg, S.O. Londen and O. Staffans, Volterra integral and functional equations, Cambridge University Press, Cambridge, 1990.

16. F. Isaia, On a nonlinear integral equation without compactness, Acta Math. Univ. Comen. 75 (2006), 233-240.

17. An existence result for a nonlinear integral equation without compactness, PanAmer. Math. J. 14 (2004), 93-106.

18. V. Istrăţescu, Fixed point theory: An introduction, mathematics and its applications, 7, Reidel Publishing Company, Boston, 1981.

19. R. Kannan, Some results on fixed points II, Amer. Math. Month. 76 (1969), 405-408.

20. Some results on fixed points III, Fund. Math. 70, (1971), 169-177.

21. A. Kufner and L.E. Persson, Weighted inequalities of Hardy type, World Scientific Publishing Co., Singapore, 2003.

22. V. Lakshmikantham and M.R.M. Rao, Theory of integro-differential equations, in Stability and control: Theory, methods and applications 1, Gordon and Breach Publishers, Philadelphia, 1995.

23. V.G. Mazya, Sobolev spaces, Springer-Verlag, Berlin, 1985.

24. J.R. Morales and E.M. Rojas, On the sum of contractive type of mappings II: Maps on different classes, J. Inequal. Appl. 2014, 208.

25. R. Morales and E. Rojas, Some results for one class of discontinuous operators with fixed points, Acta Math. Univ. Comen. 76 (2007), 149-160.

26. L. Nova, Fixed point theorems for some discontinuous operators, Pac. J. Math. 123 (1986), 189-196.

27. H. Rafeiro, Kolmogorov compactness criterion in variable exponent Lebesgue spaces, Proc. A. Razmazde Math. Inst. 150 (2009), 105-113.

28. S. Reich, Kannan's fixed point theorems, Boll. Union. Math. Ital. 4 (1971), $121-128$.

29. Remarks concerning contraction mappings, Canad. Math. Bull. 14 (1971), 121-124.

30. Remarks on fixed points, Rend. Accad. Naz. Lincei. 52 (1972), 689697.

31. B.E. Rhoades, A comparison of various definitions of contractive mappings, Trans. Amer. Math. Soc. 226 (1977), 257-290. 
32. M. Růžička, Electroreological fuids: Modeling and mathematical theory, Lecture Notes Math. 1748 (2000), 178 pages.

Departamento de Matemáticas, Universidad Nacional de Colombia, BoGotá, Colombia

Email address: recastillo@unal.edu.co

Departamento de Matemáticas, Universidad de Oriente, 6101 Cumaná, Edo. SUCRE, VENEZUELA

Email address: jcramos@udo.edu.ve

Departamento de Matemáticas, Universidad Nacional de Colombia, BoGotá, Colombia

Email address: emrojass@unal.edu.co 